\title{
Canonical approach to the finite density QCD with winding number expansion
}

\author{
Atsushi Nakamura \\ RIISE, Hiroshima University \\ Higashi-Hiroshima 739-8521, Japan \\ E-mail: nakamuradrise.hiroshima-u.ac.jp \\ Shotaro Oka \\ Institute of Theoretical Physics, Department of Physics, Rikkyo University \\ Toshima-ku, Tokyo 171-8501, Japan \\ E-mail: okasho-hatodrikkyo.ac.jp

\section{Yusuke Taniguchi*} \\ Graduate School of Pure and Applied Sciences, University of Tsukuba \\ Tsukuba, Ibaraki 305-8571, Japan \\ E-mail: tanigchidhet.ph.tsukuba.ac.jp
}

\begin{abstract}
The canonical partition function is related to the grand canonical one through the fugacity expansion and is known to have no sign problem. In this paper we perform the fugacity expansion by a method of the hopping parameter expansion in temporal direction for the lattice QCD: winding number expansion. The canonical partition function is constructed for $N_{f}=2$ QCD starting from gauge configurations at zero chemical potential. After derivation of the canonical partition function we calculate hadronic observables like chiral condensate and quark number density and the pressure at the real chemical potential.
\end{abstract}

The 32nd International Symposium on Lattice Field Theory

23-28 June, 2014

Columbia University New York, $N Y$

${ }^{*}$ Speaker. 


\section{Introduction}

The grand canonical ensemble is a difficult subject to treat in lattice QCD because of the sign problem. The canonical partition function is related to the grand canonical one through the fugacity expansion and is known to have no sign problem as will be briefly reviewed in Sec. 2 . In this paper we perform the fugacity expansion by a method of the hopping parameter expansion as is discussed in Sec. 3. The canonical partition function is evaluated for $N_{f}=2$ QCD starting from gauge configurations at zero chemical potential in Sec. 5. After derivation of the canonical partition function we study the chemical potential dependences of hadronic observables like chiral condensate and quark number density in Sec. 6.

\section{Canonical partition function}

It is a well known fact that the grand canonical ensemble and the canonical one are equivalent each other. This is shown by a simple equation to relate the grand canonical partition function $Z_{G}(\mu, T, V)$ and the canonical $Z_{C}(n, T, V)$

$$
Z_{G}(\mu, T, V)=\sum_{n=-\infty}^{\infty} Z_{C}(n, T, V) \xi^{n}, \quad \xi=e^{\mu / T}
$$

This is a so called fugacity expansion formula with the fugacity $\xi$. The inverse of this expansion is given by using the Cauchy's integral theorem

$$
Z_{C}(n, T, V)=\oint \frac{d \xi}{2 \pi i} \xi^{-n-1} Z_{G}(\xi, T, V)
$$

where we assume that the partition function $Z_{G}(\xi, T, V)$ has singularities only at $\xi=0, \infty$ corresponding to trivial points $\mu / T= \pm \infty$ and adopt an appropriate contour around the origin. Here we notice that a phase transition point $\xi_{c}$ is not a singularity of the partition function but it is rather a zero of $Z_{G}(\xi, T, V)$ in $V \rightarrow \infty$ limit (Lee-Yang zeros) and does not affect the Cauchy's integral.

Now it is free to change the contour to a unit circle $\xi=e^{i \theta}$ and the contour integral turns out to be a Fourier transformation [四]

$$
Z_{C}(n, T, V)=\int_{0}^{2 \pi} \frac{d \theta}{2 \pi} e^{-i n \theta} Z_{G}\left(e^{i \theta}, T, V\right)
$$

A standard Monte Carlo simulation is possible for the lattice QCD since the chemical potential is set to be pure imaginary $\mu / T=i \theta$ and the Boltzmann weight is real positive for two flavors.

However a difficulty of the sign problem is preserved unfortunately since there remains a frequent cancellation in plus and minus sign in a numerical Fourier transformation. In order to get the canonical partition function accurately for large quark number $n$ we need a very fine sampling of $Z_{G}\left(e^{i \theta}, T, V\right)$ in $\theta$. This requires a heavy computational cost because we need to evaluate the Dirac operator determinant for the QCD grand partition function. In this paper we shall solve this problem by a direct expansion of the Dirac determinant in terms of the fugacity. 


\section{Winding number expansion}

We consider the lattice QCD grand partition function given in the path integral form

$$
Z_{G}(\xi, T, V)=\int D U \operatorname{Det} D_{W}(\xi ; U) e^{-S_{G}(U)}
$$

where we adopt the Wilson Dirac operator

$$
\begin{aligned}
& D_{W}=1-\kappa Q, \quad Q=\sum_{i=1}^{3}\left(Q_{i}^{+}+Q_{i}^{-}\right)+e^{\mu a} Q_{4}^{+}+e^{-\mu a} Q_{4}^{-}, \\
& \left(Q_{\mu}^{+}\right)_{n m}=\left(1-\gamma_{\mu}\right) U_{\mu}(n) \delta_{m, n+\hat{\mu}}, \quad\left(Q_{\mu}^{-}\right)_{n m}=\left(1+\gamma_{\mu}\right) U_{\mu}^{\dagger}(m) \delta_{m, n-\hat{\mu}} .
\end{aligned}
$$

Both the chemical potential $e^{ \pm \mu a}$ and the hopping parameter $\kappa$ appear in front of the temporal hopping term simultaneously. The fugacity expansion of the determinant shall be given by using the hopping parameter expansion.

Instead of the determinant we consider the hopping parameter expansion of

$$
\operatorname{Tr} \log D_{W}(\mu)=\operatorname{Tr} \log (I-\kappa Q)=-\sum_{n=1}^{\infty} \frac{\kappa^{n}}{n} \operatorname{Tr}\left(Q^{n}\right)
$$

Since every term has a trace the quark hoppings should make a loop. A non-trivial chemical potential dependence appears when the quark hoppings make a loop in the temporal direction. If one of the term has a $n$ times winding loop in positive temporal direction the chemical potential dependence is $e^{\mu a n N_{t}}$ where $N_{t}$ is a temporal lattice length and this is nothing but $e^{n \mu / T}=\xi^{n}$. Counting the winding number in temporal direction for each term in (B.4) we get the winding number expansion of the TrLog

$$
\operatorname{Tr} \log D_{W}(\mu)=\sum_{n=-\infty}^{\infty} w_{n} \xi^{n}
$$

Regrouping the summation we have a fugacity expansion of the determinant [D]

$$
\operatorname{Det} D_{W}(\xi ; U)=\exp \left(\sum_{n=-\infty}^{\infty} w_{n} \xi^{n}\right)=\sum_{n=-\infty}^{\infty} z_{n}(U) \xi^{n}
$$

In this approach the fugacity dependence of the determinant or the partition function is given analytically. A numerical Fourier transformation (R.3) can be executed safely at any high precision we want. In this paper the regrouping (B.6) is done by the Fourier transformation.

The winding number expansion is done for gauge configurations generated at $\mu=0$ or purely imaginary value. This procedure corresponds to the standard reweighting method

$$
Z_{G}(\xi, T, V)=\int D U \frac{\operatorname{Det} D_{W}(\mu)}{\operatorname{Det} D_{W}\left(\mu_{0}\right)} \operatorname{Det} D_{W}\left(\mu_{0}\right) e^{-S_{G}} .
$$




\begin{tabular}{|c|c|c|c|}
\hline$\beta$ & $\kappa$ & PCAC mass & $m_{\pi} / m_{\rho}$ \\
\hline 0.9 & 0.137 & $0.17(13)$ & $0.8978(55)$ \\
1.1 & 0.133 & $0.18(19)$ & $0.9038(56)$ \\
1.3 & 0.133 & $0.088(53)$ & $0.8656(72)$ \\
1.5 & 0.131 & $0.116(39)$ & $0.8482(57)$ \\
1.7 & 0.129 & $0.168(21)$ & $1.0429(46)$ \\
1.9 & 0.125 & $0.1076(68)$ & $0.7310(95)$ \\
2.1 & 0.122 & $0.1259(11)$ & $0.833(12)$ \\
\hline
\end{tabular}

Table 1: $(\beta, \kappa)$ for the numerical simulation. The PCAC mass and $m_{\pi} / m_{\rho}$ are also given.

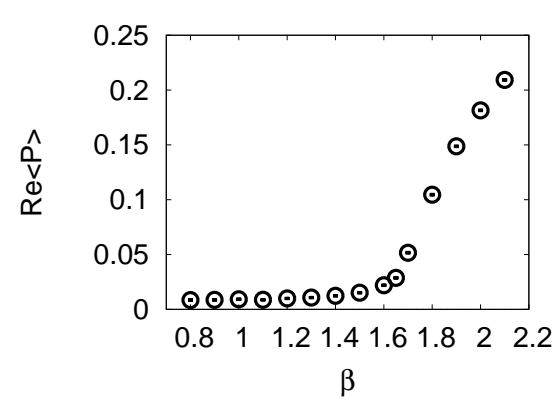

Figure 1: The Polyakov loop as a function of $\beta$.

\section{Numerical setup}

We adopt the Iwasaki gauge action and the improved Wilson fermion action with the clover term. The number of flavors is set to $N_{f}=2$ with degenerate masses. The APE stout smeared gauge link is used for those in the fermion action including the clover term. The number of smearing is four and the parameter is set to $\rho=0.1$. The clover coefficient is fixed to $c_{\mathrm{SW}}=1.1$ [目]. We adopt $8^{3} \times 4$ lattice. The numerical parameters $(\beta, \kappa)$ are given in table $\mathbb{W}$. A wide range of $\beta$ is covered from high temperature $\beta=2.1$ to low temperature 0.9 . It seems to be that both the confining and deconfining region are well covered, which is inferred from a behavior of the Polyakov loop in Fig. W. The hopping parameter is selected in order that the hopping parameter expansion works well, for which $m_{\pi} / m_{\rho}$ turns out to be 0.7 - 1.0. Maximal order of the hopping parameter expansion is set to 480 so that max winding number in temporal direction is 120 .

\section{Canonical and grand canonical partition function}

The first numerical result we get is the canonical partition function $Z_{C}(n, T, V)$. We plot $\log \left|Z_{C}\left(n_{B}, T, V\right) / Z_{C}(0, T, V)\right|$ and its phase as a function of the baryon number $n_{B}$ in Fig. $\square$. The partition function decays very rapidly with $n_{B}$ and its behavior changes drastically between $\beta=1.7$ (green) and 1.5 (orange), which may correspond to a phase transition. The phase of the partition function is consistent with zero within the error bar for every $\beta$ although only one data at $\beta=1.9$ is shown for an example.

The plot range is fixed by using the d'Alembert's convergence condition

$$
\lim _{n_{B} \rightarrow \infty}\left|\frac{Z_{C}\left(n_{B}+1\right)}{Z_{C}\left(n_{B}\right)} \xi\right|<1,
$$

${ }^{1}$ which gives the convergence radius for the fugacity $\xi$. The data at $\beta=1.9$ is plotted in right panel of Fig. 3 for example. We cut our data at $n_{\max }$ where a monotonic decrease stops indicated by vertical orange lines in the figure. The horizontal red line shows a maximal value of the fugacity expected to be within the convergence radius. By taking log the line gives our applicable limit for the baryon chemical potential $\mu_{B} / T$. For example we can discuss physics safely at $-4<\mu_{B} / T<4$ for $\beta=1.9$ with our method.

\footnotetext{
${ }^{1}$ For negative baryon number we adopt $\left|Z_{C}\left(n_{B}-1\right) / Z_{C}\left(n_{B}\right)\right|$.
} 

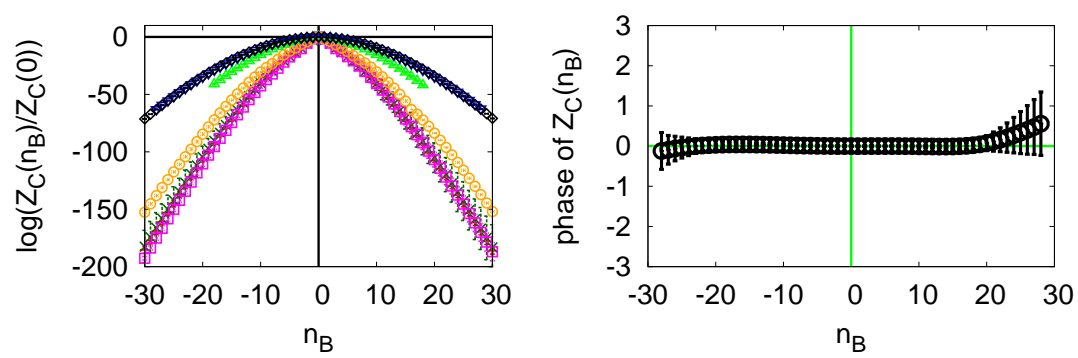

Figure 2: The canonical partition function as a function of the baryon number $n_{B}$. Left panel is the absolute value $\log \left|Z_{C}\left(n_{B}\right) / Z_{C}(0)\right|$. From the top $\beta=2.1$ (black), 1.9 (blue), 1.7 (green), 1.5 (orange), 1.3 (magenta), 1.1 (dark green), 0.9 (red). Right panel is a phase of $Z_{C}\left(n_{B}\right)$ at $\beta=1.9$.
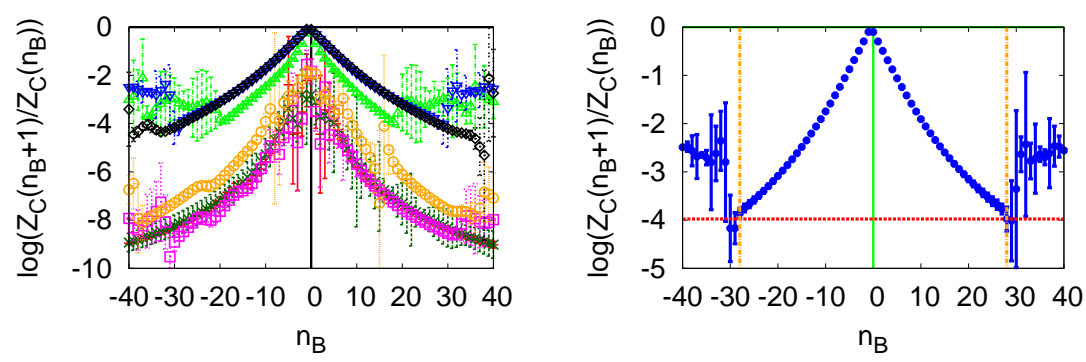

Figure 3: $\log$ plot of $\left|Z_{C}\left(n_{B}+1\right) / Z_{C}\left(n_{B}\right)\right|$ as a function of the baryon number. The color and $\beta$ correspondence is the same as in Fig. 2 for left panel. Right panel is that at $\beta=1.9$ for example.

The second physical quantity is the grand partition function. By taking summation for $-n_{\max } \leq$ $n_{B} \leq n_{\max }$ in (메) we get the grand partition function for the real chemical potential. We plot $\left(\log \left|Z_{G}(\mu, T, V) / Z_{G}(0, T, V)\right|\right) / V$ in Fig. . 1 as a function of the quark chemical potential. According to the statistical physics the logarithm of the grand partition function divided by the spatial volume is the pressure

$$
\frac{P}{T}=\frac{1}{V} \log Z_{G}
$$

The quantity plotted in Fig. 团 is the pressure $(P(\mu / T)-P(0)) / T$ in the grand canonical ensemble normalized at $\mu=0$.

\section{Hadronic observables}

In our procedure the fugacity expansion is based on the hopping parameter expansion. It may be possible to expand any hadronic operators in terms of the fugacity. We consider a fugacity expansion of a numerator of some operator VEV

$$
\langle O\rangle_{G}(\xi, T, V)=\frac{O_{G}(\xi, T, V)}{Z_{G}(\xi, T, V)},
$$




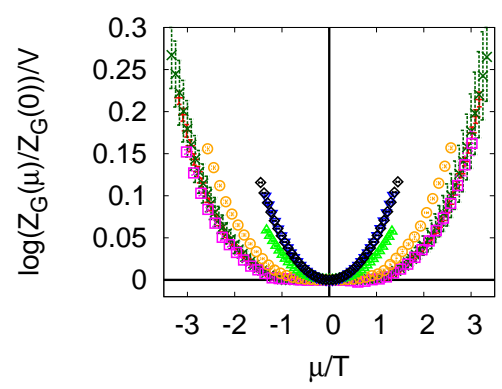

Figure 4: The grand partition function $\left(\log \left|Z_{G}(\mu, T, V) / Z_{G}(0, T, V)\right|\right) / V$ as a function of the quark chemical potential $\mu / T$. The color and $\beta$ correspondence is the same as in Fig. 2.
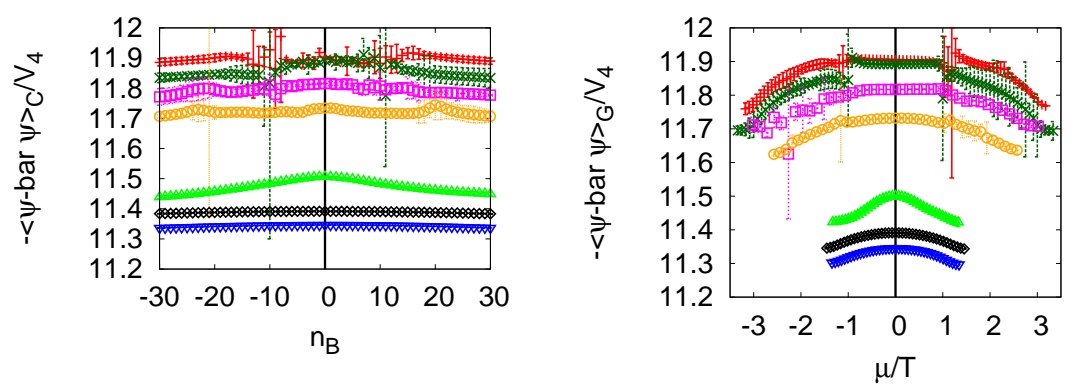

Figure 5: Left panel is the chiral condensate $\langle\bar{\psi} \psi\rangle$ in the canonical ensemble as a function of the baryon number. Right panel is that in the grand canonical ensemble as a function of the quark chemical potential $\mu / T$. The color and $\beta$ correspondence is the same as in Fig. 2.

$$
O_{G}(\xi, T, V)=\int D U\langle O\rangle_{\text {quark }}(\xi) \operatorname{Det} D_{W}(\xi ; U) e^{-S_{G}(U)}=\sum_{n=-\infty}^{\infty} O_{C}(n, T, V) \xi^{n},
$$

where $\langle O\rangle_{\text {quark }}$ is a Wick contraction of a hadronic operator $O$ in quark fields. For example we consider the chiral condensate. It is easy to expand its Wick contraction in terms of the hopping parameter

$$
\langle\bar{\psi} \psi\rangle_{\text {quark }}=-\operatorname{tr}\left(\frac{1}{D_{W}}\right)=-\operatorname{tr}\left(\frac{1}{1-\kappa Q}\right)=\sum_{m=0}^{\infty} \kappa^{m} \operatorname{tr} Q^{m}=\sum_{n=-\infty}^{\infty} o_{n}(U) \xi^{n} .
$$

Counting the winding number in temporal direction we get the last equality. Multiplying the determinant contribution (B.6) we apply the same regrouping procedure in Sec. B] and get the fugacity expansion (6.2).

Once we have two coefficients $Z_{C}(n, T, V)$ and $O_{C}(n, T, V)$ the VEVs of the operator with the canonical and the grand canonical ensemble are available. The canonical ensemble VEV is given by taking the ratio of two coefficient

$$
\langle O\rangle_{C}(n, T, V)=\frac{O_{C}(n, T, V)}{Z_{C}(n, T, V)} .
$$

The results for the chiral condensate $-\langle\bar{\psi} \psi\rangle_{C}$ are given in the left panel of Fig. [ 1 as a function of the baryon number. A VEV in the grand canonical ensemble is given by taking fugacity summation 


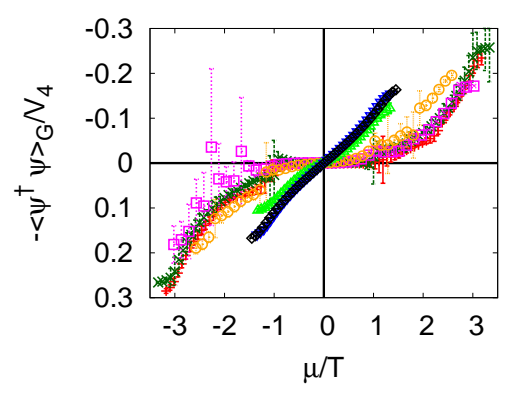

Figure 6: Quark number density $-\left\langle\psi^{\dagger} \psi\right\rangle_{G}$ as a function of the quark chemical potential.

with real chemical potential

$$
\langle O\rangle_{G}(\xi, T, V)=\frac{\sum_{n=-n_{\max }}^{n_{\max }} O_{C}(n, T, V) \xi^{n}}{\sum_{n=-n_{\max }}^{n_{\max }} Z_{C}(n, T, V) \xi^{n}} .
$$

The chiral condensate $-\langle\bar{\psi} \psi\rangle_{G}$ is given in the right panel of Fig. $\square$ as a function of the quark chemical potential $\mu / T$. The condensate in the figure is a bare quantity without renormalization. Since we adopted the Wilson fermion we have an additive correction for $\langle\bar{\psi} \psi\rangle$, which is not subtracted in this paper.

From the right panel of Fig. 1 the chiral restoration phase transition at finite chemical potential seems to be clear. A relatively large value around $\mu / T=0$ decreases at large chemical potential and a fall occurs rapidly at a narrow region. The would-be transition parameter $\mu / T$ becomes larger for lower temperature.

In Fig. 6 the quark number density $-\left\langle\psi^{\dagger} \psi\right\rangle_{G}$ in the grand canonical ensemble is plotted as a function of the quark chemical potential $\mu / T$.

\section{Conclusion}

In this paper we performed the fugacity expansion of the grand partition function by using the hopping parameter expansion. This procedure seems to be valid for baryon numbers around $n_{B} \sim 30$ for $8^{3} \times 4$ lattice. The method is also applied to the numerator of VEVs of hadronic operators. Taking summation we get a VEV at the real chemical potential. As an example we evaluate the chiral condensate and show a phase transition like behavior at high chemical potential for low temperature region.

This work is done for $\mathrm{Zn}$ Collaboration. This work is supported in part by Grants-in-Aid of the Ministry of Education (Nos. 26610072. 24340054, 22540265). This work is in part based on Bridge++ code (http://suchix.kek.jp/bridge/Lattice-code/).

\section{References}

[1] A. Hasenfratz and D. Toussaint, Nucl. Phys. B 371, 539 (1992).

[2] A. Li, A. Alexandru, K. F. Liu and X. Meng, Phys. Rev. D 82, 054502 (2010)

[3] Y. Taniguchi, PoS LATTICE 2012, 236 (2012) 